\title{
¿DEPENDE LA SATISFACCIÓN ESTUDIANTIL DE LA CALIDAD REAL DE LA FACULTAD O DE LA PERCEPCIÓN INDIVIDUAL DE ESTA? ANÁLISIS COMPARATIVO ENTRE FACULTADES PRIVADAS Y PÚBLICAS
}

\section{DOES STUDENT SATISFACTION DEPEND ON THE ACTUAL QUALITY OF THE FACULTY OR ON THE PERCEPTION OF THE STUDENT? COMPARATIVE ANALYSIS BETWEEN PUBLIC AND PRIVATE FACULTIES}

Pérez Zamora, L.A., López López, M.C., Couto Olivares, J.M. y Navarro Luque, G. Universidad de Granada

Fecha de recepción: 28 de mayo de 2012

Fecha de aceptación: 11 de junio de 2012

Localizador: http:www.ugr.es/local/miguelgr/ReiDoCrea-Vol.1-Art.4-Perez-Lopez-Couto-Navarro.pdf

\section{Resumen}

El objetivo de este estudio es conocer la satisfacción estudiantil en relación a las universidades públicas y privadas. Es un estudio correlacional, realizado en la Universidad de Granada en la escuela universitaria de magisterio La Inmaculada y en la de facultad de ciencias de la educación. Participaron 111 alumnos entre ambas facultades que completaron un cuestionario sobre satisfacción estudiantil. Los principales resultados exponen que a pesar de no obtener diferencias significativas en la satisfacción global, sí lo hacen en distintos aspectos, obteniendo mayor satisfacción los estudiantes de la Facultad privada, en competencias profesionales y servicio administrativo, en comparación con los de la pública que obtienen mayores puntuaciones en satisfacción con el ambiente en la facultad y la biblioteca.

Palabras Clave: Satisfacción estudiantil, universidad pública, universidad privada.

\section{Abstract}

The aim of this study is to determine student satisfaction in relation to public and private universities. The study is correlational, and was carried out in Granada, at the University School of Education of La Inmaculada and at the Faculty of Education. 111 students from both faculties completed a questionnaire about student satisfaction. The main results of this study show that despite there not being significant differences in the overall mean, there were differences in some aspects as the private university students were more satisfied with respect to professional skills, and admininistrative service than the public university students who obtained higher scores in satisfaction with the atmosphere of the faculty and of the library.

Key words: Student satisfaction, public university; private university. 


\section{INTRODUCCIÓN}

Con el siguiente estudio se pretende comprobar cuáles son las variables relacionadas con la satisfacción estudiantil, y cómo afecta la naturaleza del centro de enseñanza a dicha percepción, por lo tanto, sería interesante comenzar exponiendo que entenderemos por satisfacción estudiantil, aquella percepción que surge en el alumno cuando la universidad o centro educativo cubre sus necesidades (Flores Balboza, 2005).

Desde la literatura se cree que las necesidades de los alumnos se perciben mas cubiertas por aquellos que acuden a centros de enseñanza privados, que aquellos que van a los centros públicos. Esto podría estar influenciado por sus intereses y expectativas, desde la literatura se defiende que estas son distintas, esto es, los alumnos de centros de naturaleza privada o concertada tienen mayor aspiración educativa que los alumnos de los centros públicos (Fernández Enguita, 2008).

La satisfacción del estudiante refleja la eficiencia de los diversos aspectos que componen el día a día de su experiencia educacional, estando esta relacionada con el rigor de los cursos, con las interacciones con su profesor y los otros estudiantes, con la justicia con que se le evalúa, etc., así como con las instalaciones y equipamiento que apoyan dichas interacciones (Flores Balboza, 2005).

En un estudio de la universidad de Lima, sobre la satisfacción en docentes, se encuentra que la satisfacción en los docentes de las facultades privadas es mayor que la de los docentes de las facultades públicas, tanto por factores extrínsecos como intrínsecos, éste además de explicar que esto puede ser debido a las infraestructuras y a las características de la organización, expone que también puede influir el hecho de la percepción que se tenga de dicha universidad, influyendo aquí las expectativas (Álvarez Flores, 2007).

Una vez expuesto esto, es interesante extrapolarlo al polo de los alumnos, si los profesores ven como de mayor calidad el centro privado y por lo tanto les agrada más trabajar allí, debido a una percepción previa a dicho trabajo, podríamos pensar que los alumnos pueden estar más satisfechos también por una percepción previa de la facultad, esto es, si ellos escogieron entrar en una facultad privada aún teniendo que pagar, sería debido a que las expectativas que tenían eran que la facultad privada les podría aportar algo que la pública no, y el hecho de aceptar pagar por asistir a ella, elevaría dicho compromiso, ya que se pagaría por algo que aportase calidad, ya que el hecho de pagar llevaría consigo la creencia de que se paga por obtener algo mejor de lo que se puede obtener sin hacerlo, quedando justificada así la necesidad de pagar por asistir a la facultad privada existiendo la universidad pública.

Estaríamos recurriendo a la profecía autocumplida, si esperamos que dicha universidad sea buena de antemano, será más fácil que nos parezca buena una vez estemos en ella. Los alumnos de las universidades privadas, si están dispuestos a pagar, esperan encontrar algo que se les escaparía en la pública. Según esta teoría, lo importante aquí son las expectativas creadas de antemano, las cuales van a contribuir a las percepciones posteriores. Esto se crea mediante el siguiente desarrollo, se formas las expectativas, se establecen los medios para la realización de éstas, y por último se confirman nuestras expectativas (Burón, 2005).

Este proceso depende de las características personales y de la percepción de la realidad de cada uno, dependiendo esto de la atención a unos aspectos y no otros, lo cual estará relacionado con el sistema de creencias e ideología de cada persona (Pichardo, García Berbén, De la Fuente y Justicia, 2007). 
Por otra parte, es interesante hablar de disonancia cognitiva, esta viene a significar un conflicto generado en el sistema de creencias y emociones, o un comportamiento de la persona que entra en conflicto con las creencias, esto es, la disonancia sería la percepción de incompatibilidad de dos cogniciones simultáneas, en nuestro caso ésta se daría en los alumnos de las facultades privadas si pagasen en la facultad y además pensasen que es de peor calidad (Festinger, 1954).

Por lo tanto, atendiendo a ambas teorías cabría suponer que los alumnos de las facultades privadas puntuarán más alto en satisfacción que los alumnos de las facultades públicas, por un lado, los alumnos que entren en las facultades privadas, lo harán con una idea previa de calidad de esa facultad, por la cual merece la pena pagar, generando esto unas elevadas expectativas aumentando su percepción de satisfacción. El hecho de pagar, si no se percibiese como algo necesario y acertado, generaría un conflicto en el sistema de creencias, por lo que es de suponer que tienen la creencia de recibir a cambio una educación de mejor calidad, frente a los que no pagan.

A lo mencionado anteriormente, hay que añadirle la naturaleza del estudiante, la satisfacción puede estar influenciada por otras variables como son el plan docente al que el alumno esta acogido, por ejemplo actualmente en la Universidad de Granada se acogen dos planes, en los primeros cursos de las carreras esta instaurado el plan Bolonia, mientras que en los cursos más avanzados aún perdura el plan 2000 (Flores Balboza, 2005).

Uno de los factores más diferenciadores entre ambos a día de hoy podría ser la libertad de elegir las asignaturas, algo que se da en el plan 2000 pero no en el plan Bolonia, y la duración de las carreras, ya que todas pasan a ser de 4 años. Para comprobar si verdaderamente esto es así, se escogerán parte de la muestra de alumnos del plan 2000 y la otra parte de alumnos del plan nuevo.

Con esta investigación se pretende comprobar si, efectivamente la naturaleza de la facultad influye en la satisfacción, aumentándola en el caso de la privada, y por otro, como afecta a dicha satisfacción el plan de estudios que siguen los alumnos y que variables son las que generan dicha satisfacción. Para ello, se realizó la investigación tanto con alumnos de facultades privadas como públicas, y a su vez de ambos planes. Se medirá mediante un cuestionario de satisfacción que hace referencia a 9 áreas relacionadas con la facultad, éstas son, competencias profesionales, personal docente, ambiente en la facultad, biblioteca, servicio de ordenadores, cafetería, servicio académico, servicio administrativo y actitudes personales y sociales en la facultad.

\section{MÉTODO}

\section{Participantes}

Para la realización del siguiente estudio, se selecciono una muestra de 111 sujetos, de los cuales el $46,8 \%$ eran hombres y el $53.2 \%$ mujeres, con una media de edad de 21 años (DT= 2'845).

Esta muestra fue tomada en dos facultades, donde el $48,6 \%$ era de naturaleza pública (Facultad de Ciencias de la Educación, Universidad de Granada), y el 51,4\% de naturaleza privada (Escuela Universitaria de Magisterio la Inmaculada, Granada) $51,4 \%$. 
También se controló el plan de estudios al que estaban sujetos los alumnos, esto es, $50.5 \%$ de alumnos de diplomatura en magisterio, y el $49,5 \%$ de alumnos de grado en magisterio, repartidos de manera equitativa en ambas facultades.

\section{Diseño}

Se trata de un estudio correlacional donde se pretende ver la relación entre la naturaleza de la facultad (VI1) con la satisfacción estudiantil (VD) por un lado, y por otro si la satisfacción también está relacionada con el plan de estudios al que están sujetos los alumnos (VI2).

\section{Instrumentos}

Para medir la satisfacción se utilizó una adaptación del cuestionario de satisfacción estudiantil de Flores (2005).

Este cuestionario divide la satisfacción en 9 áreas relacionadas con la facultad, que son competencias profesionales, personal docente, ambiente en la facultad, biblioteca, servicio de ordenadores, cafetería, servicio académico, servicio administrativo, y actitudes personales y sociales.

La satisfacción se medía en una escala tipo Likert de 1 a 4, siendo 1 nada satisfecho y 4 muy satisfecho.

\section{Procedimiento}

Este estudio se realizó mediante el método de encuesta. Se seleccionaron al azar sujetos en ambas facultades, controlando que hubiese estudiantes de ambos planes de estudios.

Se le pedía al participante que indicase de una manera anónima cómo de satisfecho se encontraba con distintos aspectos de su facultad.

\section{RESULTADOS}

Media global de satisfacción en universidades públicas y privadas.

\begin{tabular}{lll}
\hline & MEDIA & DESVIACIÓN TÍPICA \\
\hline PRIVADA & 2,68 & 0,46 \\
PUBLICA & 2,73 & 0,29 \\
\hline
\end{tabular}

Aunque la puntuación de satisfacción total salió más alta en la pública, se realizó la prueba T y mostró que las diferencias no eran significativas.

Tras la realización de un análisis de correlaciones, los resultados mostraron que sí existen diferencias significativas entre ambas facultades en algunos de los ámbitos que medía el cuestionario, tales como, competencias profesionales, ambiente en la facultad, biblioteca y servicio administrativo, los resultados los describimos a continuación. 


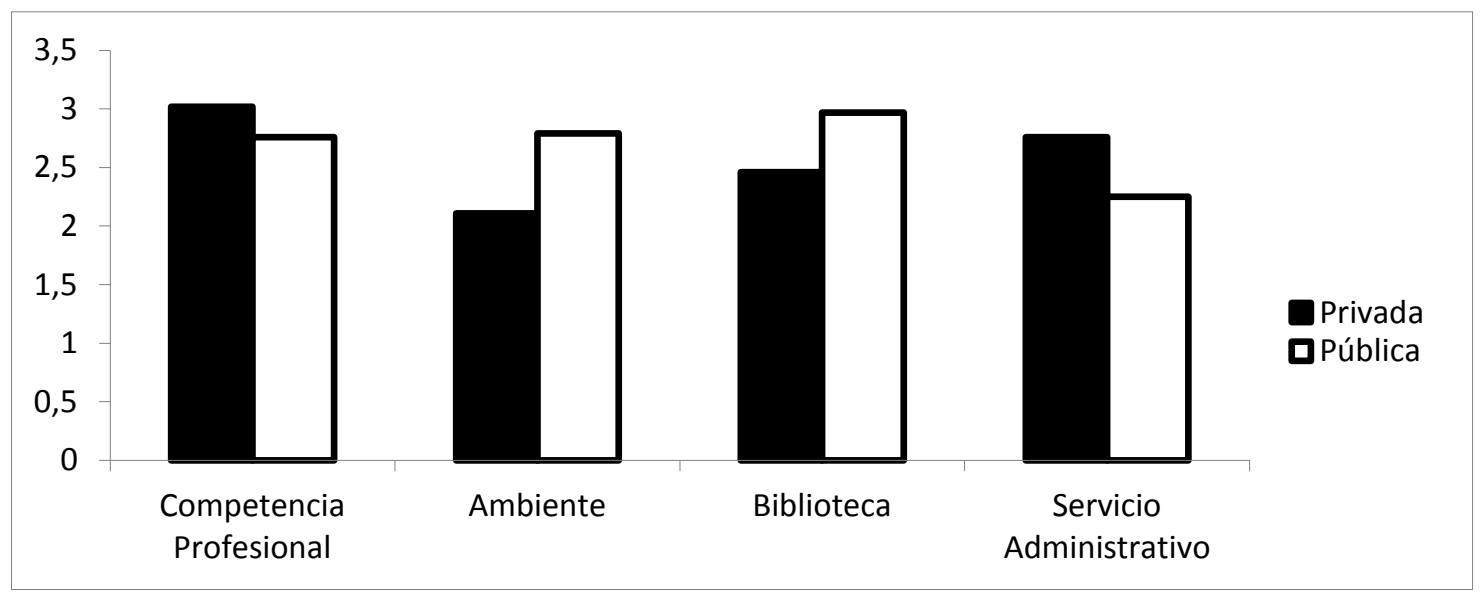

Gráfica1. Diferencias de satisfacción en Universidades públicas y privadas según diferentes ámbitos.

Como se observa en la gráfica 1 , los resultados obtenidos fueron:

Mayor satisfacción en la facultad privada en competencias personales y servicio administrativo, con una puntuación de 3,02 frente a los 2,76, $t(111)=3,118 ; p=.002$, de la pública, y 2,76 frente a $2,25, t(111)=3,186 ; p=.002$, respectivamente.

Mayor satisfacción en la facultad pública en ambiente y biblioteca, con una puntuación de 2,79 frente a $2,11, t(111)=-6,126 ; p<.001$ frente a la privada, y 2,97 frente a 2,47, $t(111)=-4,55 ; p<.001$, respectivamente.

Se realizó la prueba T y se comprobó la significación de las diferencias anteriormente nombradas.

Después se pasó a analizar los resultados en función del plan de estudios, comenzaremos con la Facultad privada.

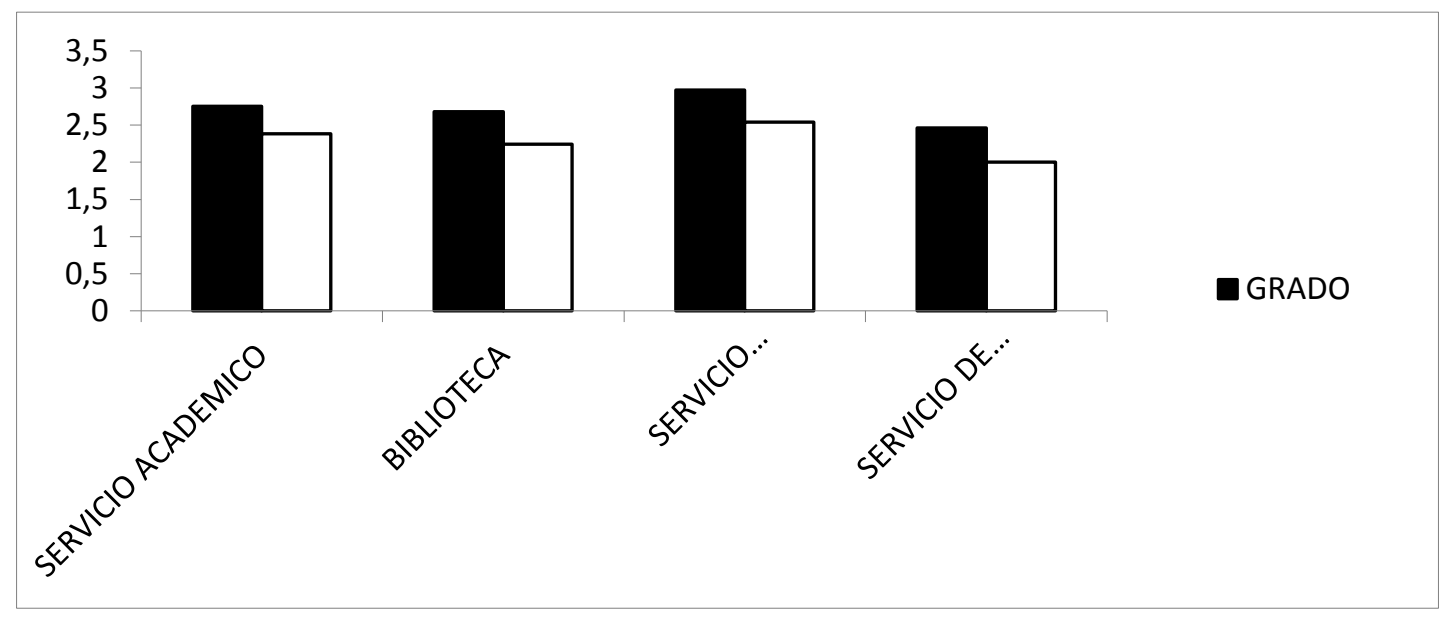

Gráfica 2. Diferencias por plan de estudios en distintos ámbitos de satisfacción de la Facultad privada. 
El análisis de los datos muestra que en general los alumnos de grado están ligeramente más satisfechos con aspectos de la facultad, con una media global de 2,79 , que los alumnos de diplomatura, cuya media global es de 2,57, $t(57)=-1,86 ; p=$ .068 .

Se realizó una prueba $\mathrm{T}$ y se comprobó que hay diferencias significativas en ciertos ámbitos, los alumnos de grado obtienen puntuaciones más altas en satisfacción en las áreas de servicio académico (2,75 vs. 2,38), $t(57)=-2,664 ; p=.010$, biblioteca $(2,68$ vs. $2,24), \mathrm{t}(57)=-2,59 ; p=.012$, servicio administrativo (2,97 vs. 2,54), $t(57)=-2,093 ; p=$ .041 , y servicio de ordenadores (2, 46 vs. 2$), t(57)=-2,462 ; p=.017$.

También se quiso ver las diferencias entre los diferentes planes de estudios tanto en la Universidad pública como en la privada.

Las diferencias encontradas con respecto a la facultad pública fueron las siguientes:

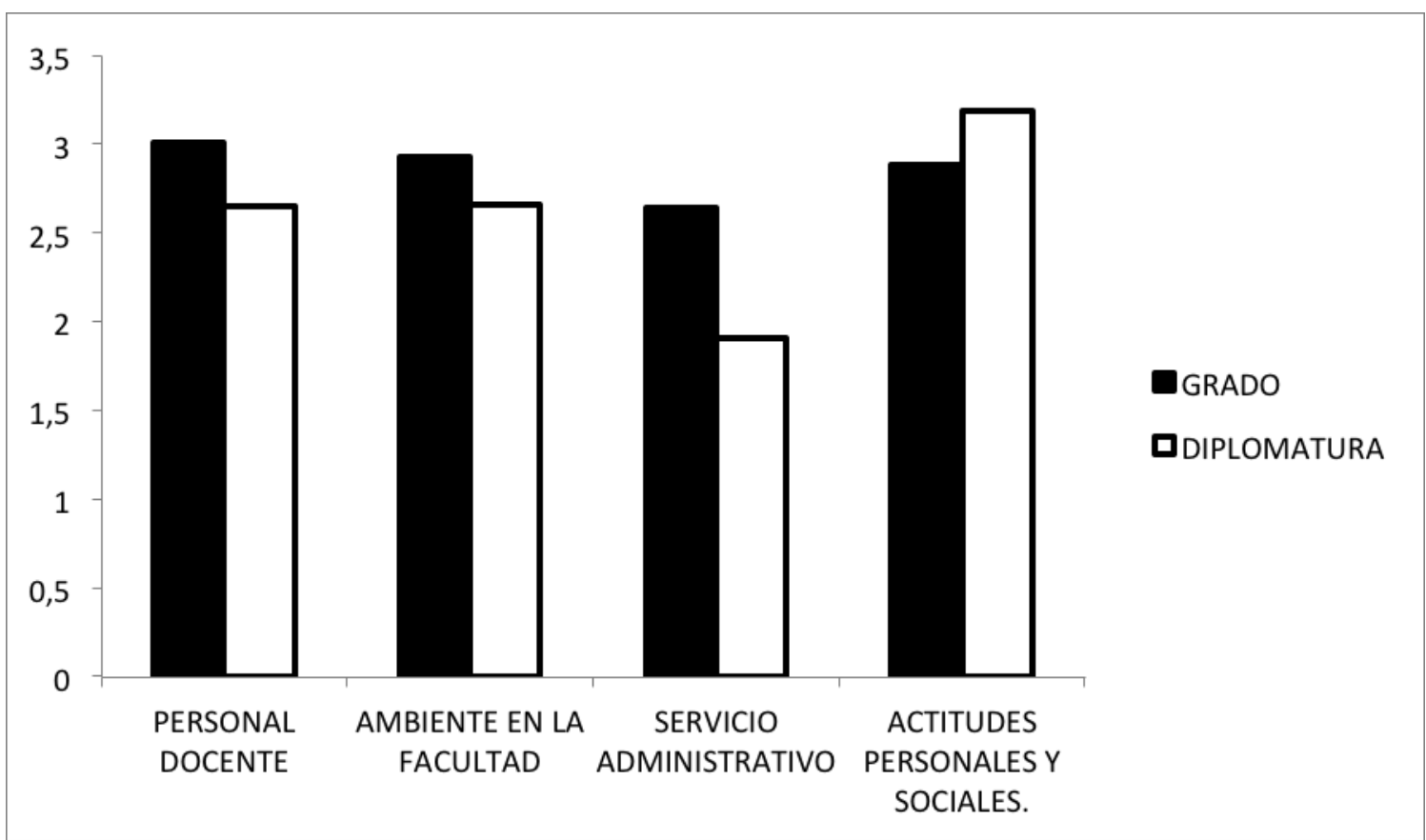

\section{Gráfica 3. Diferencias por plan de estudios en distintos ámbitos de satisfacción de la Facultad pública.}

Con respecto a la facultad pública, los alumnos de grado suelen estar más satisfechos en general (con medias globales de 2,84 alumnos de grado y 2,64 alumnos de diplomatura), $t(54)=-2,59 ; p=.012$, con aspectos relacionados con la facultad, $y$ concretamente con las áreas de personal docente $(3,01$ vs. 2,65$), t(54)=-2,964 ; p=$ .005 , ambiente en la facultad (2,93 vs. 2,66$), t(54)=-2,622 ; p=.011$, servicio administrativo (2,64 vs. 1,91$), \mathrm{t}(54)=-3,207 ; p=.002$.

Sin embargo en el área de actitudes personales y sociales son los alumnos de diplomatura los que se encuentran más satisfechos que los alumnos de grado, con puntuaciones medias de 3,19 alumnos de diplomatura, 2,88 alumnos de grado, $t(54)=$ 2,$645 ; p=.011$. 
Por último se analizaron las diferencias de sexo entre las facultades privada y pública, a continuación presentamos los datos obtenidos:

- Facultad privada:

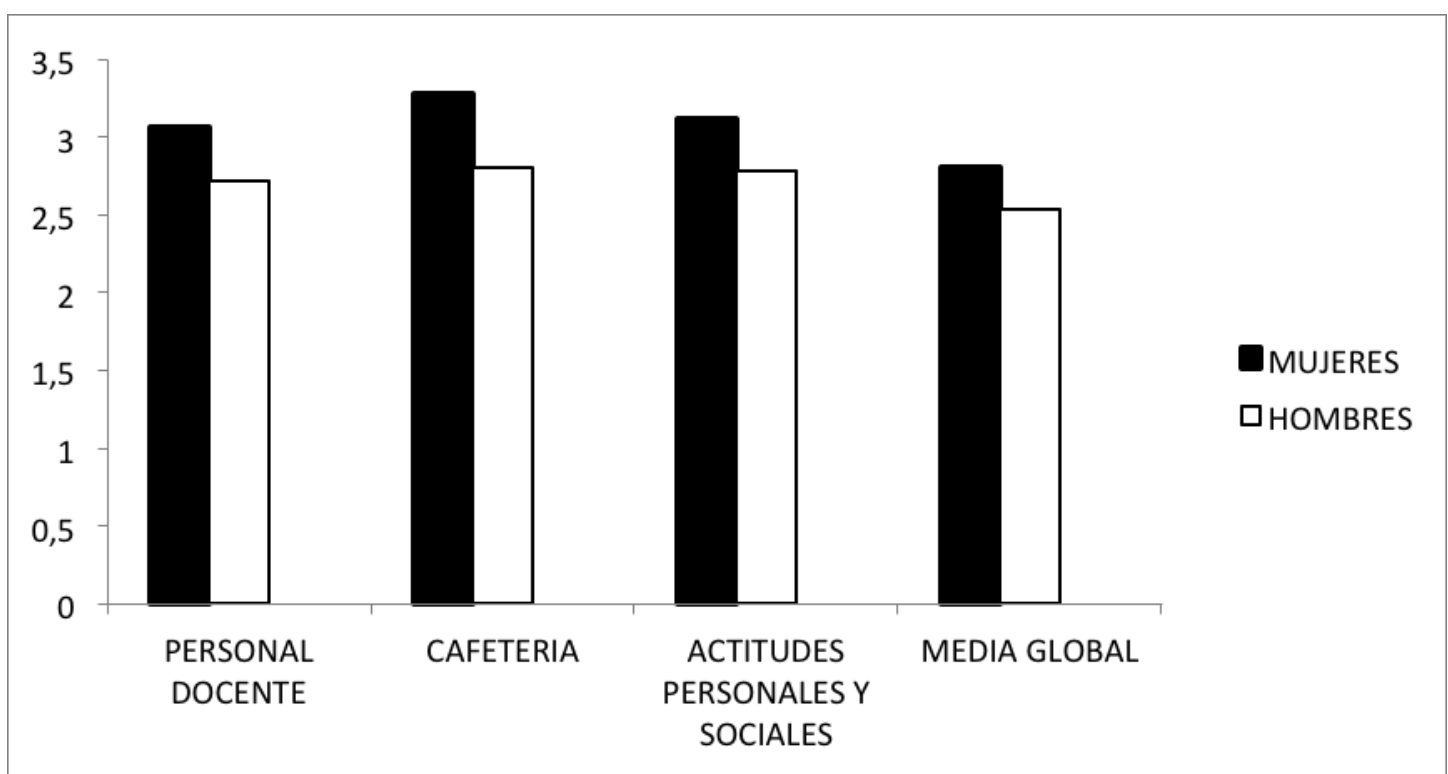

\section{Gráfica 4. Diferencias de género en distintos ámbitos de satisfacción en la facultad privada.}

Como podemos ver, hay diferencias significativas tanto en la media global $(2,8$ mujeres vs. 2,53 hombres), $t(57)=-2,19 ; p=.033$; como en las áreas de personal docente (3,06 mujeres vs. 2,72 hombres), $t(57)=-2,167 ; p=.351$; cafetería $(3,27$ mujeres vs. 2,8 hombres), $t(57)=-2,248 ; p=.029$ y actitudes personales y sociales $(3,11$ mujeres vs. 2,53 hombres $), t(57)=-2,215 ; p=.031$. Lo que indica que las mujeres están más satisfechas en general.

\section{- Facultad pública:}

Al realizar la prueba $T$ sólo se encontraron diferencias significativas en el grado de satisfacción correspondiente al área de ambiente en la facultad, obteniendo las mujeres una puntuación de 2,94 y los hombres una puntuación de 2,64, $t(54)=-3,115$; $p=.003$.

\section{DISCUSIÓN}

El estudio de la satisfacción estudiantil es importante porque ésta es el reflejo de la percepción subjetiva del alumno acerca de la cobertura de sus necesidades como estudiante (Flores Balboza, 2005).

Para la realización de este estudio se partió de la hipótesis de que los alumnos de las facultades privadas están más satisfechos que los de la facultad pública. Para su planteamiento se recurrió a la teoría de la disonancia cognitiva de Festinger (1958) y la teoría de la profecía autocumplida de Merton (1957).

A la vista de los resultados, se observa que la satisfacción es mayor para los alumnos de la Facultad privada en aspectos como las competencias profesionales y el servicio 
administrativo, aunque la diferencia en la satisfacción global no sea estadísticamente significativa.

Sí se obtienen por otro lado resultados significativos e interesantes al comparar la satisfacción entre diferentes ámbitos (recuérdese que el cuestionario medía 9 ámbitos relacionados con la satisfacción estudiantil). Al compararla en los diferentes ámbitos entre los alumnos de ambas facultades (públicas y privadas) se encontró que la satisfacción es mayor significativamente en los ámbitos de competencias profesionales y servicio administrativo para los alumnos de la Facultad privada, y en el caso de los alumnos de la pública, sus puntuaciones eran mayores para biblioteca y ambiente en la Facultad.

Se cree que tal vez el dato interesante aquí es la diferencia encontrada en las competencias profesionales, ya que éstas dependen de la percepción de cada uno y no tanto del servicio prestado por parte de la facultad como sucede en el caso concreto de la biblioteca, donde puede que sí influya la calidad de ésta en la satisfacción de los alumnos (Fernández Enguita, 2008).

Otro planteamiento de ésta investigación fue la influencia del plan de estudios. En la facultad privada los resultados muestran que los alumnos de grado están por lo general más satisfechos que los de la diplomatura, dichos resultados se podrían explicar quizás por el trato que se le da a los alumnos de grado, siendo distinto al de los alumnos de diplomatura, y esto influya en la satisfacción, pero también podría ser que dicha satisfacción sea más alta en alumnos de grado por el tiempo que llevan en la facultad con respecto a los de diplomatura (2 cursos de diferencia) (Pichardo et al. 2007).

Con respecto a la pública, los alumnos de grado suelen estar más satisfechos, salvo en el ámbito de actitudes personales y sociales, quienes son los alumnos de la facultad pública de la diplomatura los que se encuentran más satisfechos con este ámbito de toda la muestra.

Este dato puede indicar, que los alumnos de los planes antiguos de las Universidades públicas, estén desarrollando unas competencias que se escapan del resto de los alumnos, quizás esto se deba a un compromiso diferente por parte de dichos alumnos, el cual puede estar influido por factores como la extinción de las asignaturas, la dependencia de una beca y la autonomía a la hora de decidir su formación, al contrario de lo expuesto en otras investigaciones, donde se cree que son los alumnos de la facultad privada los que más desarrollan esta habilidad, hablaríamos aquí de un factor de maduración, quizás importantísimo para la formación de una persona, tanto a nivel profesional como personal (Chamorro de Saro, 2009).

Otra diferencia significativa encontrada ha sido el nivel de satisfacción en relación con el género, estando las mujeres en la facultad privada más satisfechas que los hombres, creemos que este dato quizás no sea tan significativo porque la muestra de la facultad privada era mayoritariamente mujeres en los alumnos de Grado y hombres en los de diplomatura, por lo tanto, esto puede ser un reflejo de una mayor satisfacción en los alumnos de grado y esta no dependa tanto del género.

Como posibles limitaciones podríamos señalar que quizás la facultad no sea la apropiada, ya que preguntamos el motivo por el que estaban allí, y el 89,47\% de los sujetos de la facultad privada realizan allí sus estudios porque no pudieron entrar en otra. Por lo tanto, como posible investigación futura, quizás escoger una facultad privada donde la gente quiera pagar por entrar esperando encontrar algo mejor. También es posible que esto se vea influido por el nivel económico de Andalucía, 
donde no se solicitan tanto las facultades privadas como puede suceder con otras comunidades autónomas.

\section{BIBLIOGRAFÍA}

Álvarez Flores, D. (2007). Satisfacción y fuentes de presión laboral en docentes universitarios. Periódico electrónico de psicología, (20). Recuperado de: http://pepsic.bvsalud.org/scielo.php?pid=S156061392007000100004\&script=scl arttext.

Chamorro de Sarro, M. (2009). Autonomia universitária. Revista Española de Educación Física y Deporte, 33-39 (13).

Fernández Enguita, M. (2008). Escuela pública y privada: la segregación rampante. Profesorado. Revista de Currículum y Formación del Profesorado, 12(2).

Festinger, L. (1954). A theory of social comparison processes. Human Relations, 7(2) 117-140.

Flores Balboza, J. (2005). Encuesta sobre la Satisfacción Estudiantil. Lima.

Merton, R. K. (1957). Social theory and social structure. Nueva York: Free Press

Pichardo, M. C., García- Berbén, A. B., De la Fuente, J. y Justicia, F. (2007). El estudio de las expectativas en la universidad: análisis de trabajos empíricos y futuras líneas de investigación. Revista electrónica de investigación educativa, 1-16, 9(1). 